\title{
Research on Technologies of Spatial Configuration Information Retrieval
}

\author{
Haibin Sun \\ College of Information Science and Engineering, \\ Shandong University of Science and Technology, \\ Qingdao 266510, China \\ Offer_sun@hotmail.com
}

\begin{abstract}
The problem of spatial configuration information retrieval is a Constraint Satisfaction Problem (CSP), which can be solved using traditional CSP algorithms. But the spatial data can be reorganized using index techniques like R-tree and the spatial data are approximated by their Minimum Bounding Rectangles (MBRs), so the spatial configuration information retrieval is actually based on the MBRs and some special techniques can be studied. This paper studies the mapping relationships among the spatial relations for real spatial objects, the corresponding spatial relations for their MBRs and the corresponding spatial relations between the intermediate nodes and the MBRs in R-tree.
\end{abstract}

\section{Introduction}

Spatial configuration retrieval is an important research topic of content-based image retrieval in Geographic Information System (GIS), computer vision, and VLSI design, etc. A user of a GIS system usually searches for configurations of spatial objects on a map that match some ideal configuration or are bound by a number of constraints. For example, a user may be looking for a place to build a house. He wishes to have a house A north of the town that he works, in a distance no greater than $10 \mathrm{~km}$ from his child's school B and next to a park C. Moreover, he would like to have a supermarket D on his way to work. Under some circumstances, the query conditions cannot be fully satisfied at all. The users may need only several optional answers according to the degree of configuration similarity. Of the configuration similarity query problem, the representation strategies and search algorithms have been studied in several papers $12[3]$.

In the real world, spatial data often have complex geometry shapes. It will be very costly if we directly to calculate the spatial relationships between them, while much invalid time may be spent. If $N$ is the number of spatial objects, and $n$ the number of query variables, the total number of possible solutions is equal to the number of $n$-permutations of the $N$ objects: $N ! /(N-n)$ ! . Using Minimum Bounding Rectangles (MBRs) to approximate the geometry shapes of spatial objects and calculating the relations between rectangles will reduce the calculation greatly. So we can divide the spatial configuration retrieval into two steps: firstly the rectangle combinations for which it is impossible to satisfy the query 
conditions will be eliminated, and then the real spatial objects corresponding to the remaining rectangle combinations will be calculated using computational geometry techniques. To improve the retrieval efficiency, the index data structure which is called $\mathrm{R}$-tree 4 or the variants $\mathrm{R}+$-tree $\left[5\right.$ and $\mathrm{R}^{*}$-tree 6 can be adopted.

The next section takes topological and directional relations as examples to study the mapping relationships between the spatial relationships for MBRs and the corresponding relationships for real spatial objects; the last section concludes this paper.

\section{Spatial Mapping Relationships}

This paper mainly concerns the topological and directional relations for MBRs and the corresponding spatial relationships for real spatial objects. The ideas in this paper can be applied to other relationships such as distance and spatiotemporal relations, etc.

\subsection{Topological Mapping Relationships}

This paper focuses on RCC8 [7] (see Fig[1) relations and studies the mapping relationship between the RCC8 relations for real spatial objects and the RCC8 relations for the corresponding MBRs. Let $\mathrm{p}$ and $\mathrm{q}$ be two real spatial objects, p' and q' be their corresponding MBRs. If the spatial relation between $\mathrm{p}$ and $\mathrm{q}$ is PO (Partly Overlap), then the possible spatial relation between p' and q' is PO(Partly Overlap) or TPP (Tangential Proper Part) or NTPP (Non-Tangential Proper Part) or EQ (Equal) or TPPi (inverse of Tangential Proper Part) or NTPPi (inverse of Non-Tangential Proper Part) which can be denoted by the
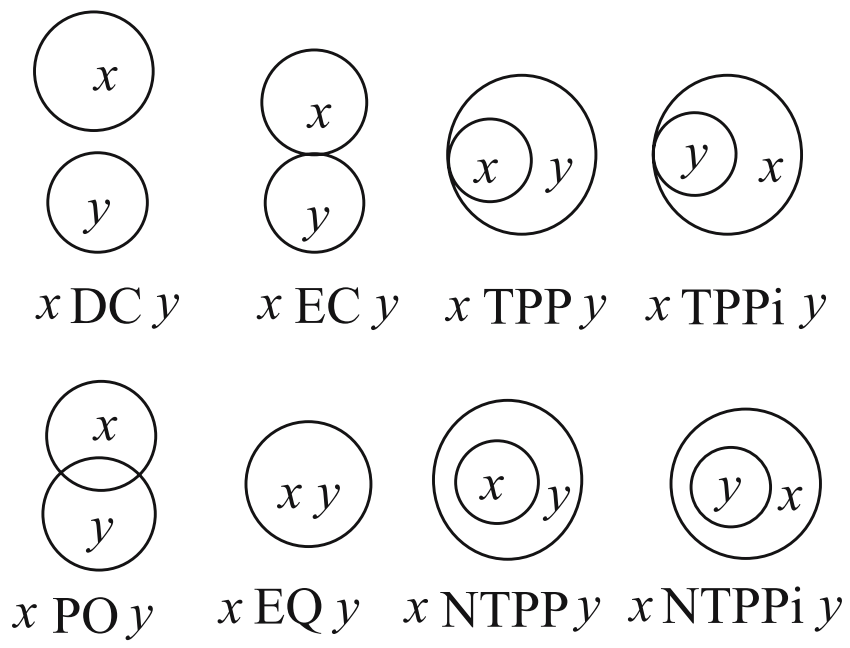

Fig. 1. Two-dimensional examples for the eight basic relations of RCC8 
disjunction form $\operatorname{PO}\left(p^{\prime}, q^{\prime}\right) \operatorname{TPP}\left(p^{\prime}, q^{\prime}\right) \operatorname{NTPP}\left(p^{\prime}, q^{\prime}\right) \operatorname{EQ}\left(p^{\prime}, q^{\prime}\right) \operatorname{TPPi}\left(p^{\prime}, q^{\prime}\right)$ $\operatorname{NTPPi}\left(p^{\prime}, q^{\prime}\right)$. To use R-tree to improve the efficiency of the spatial configuration retrieval, the topological relations in the query condition should first be transformed to the corresponding topological relations for the MBRs, which can be used to eliminate the rectangle combinations that cannot fulfill the constraints from the leaf nodes in the R-tree. The intermediate nodes in the R-tree can also be used to fast the retrieval process. Let p" be the rectangle that enclose p', i.e. the parent node of leaf node p' in the R-tree, which is called intermediate node. Given the spatial relation between p' and q', the spatial relation between p" and q' can be derived. For example, from the spatial relation $\operatorname{TPP}\left(p^{\prime}, q^{\prime}\right)$, the spatial relation $\operatorname{PO}\left(p^{\prime \prime}, q^{\prime}\right) \operatorname{TPP}\left(p^{\prime \prime}, q^{\prime}\right) \operatorname{EQ}\left(p^{\prime}, q^{\prime}\right) \operatorname{TPPi}\left(p^{\prime \prime}, q^{\prime}\right) \operatorname{NTPPi}\left(p^{\prime \prime}, q^{\prime}\right)$ can be obtained. It is very interesting that the parents of the intermediate nodes also have the same property. Table 1 presents the spatial relations between two real spatial objects, the possible spatial relations that their MBRs satisfy and the possible spatial relations between the corresponding intermediate node and the MBR.

Table 1. The spatial relations between two real spatial objects, the possible spatial relations that their MBRs satisfy and the possible spatial relations between the corresponding intermediate node and the MBR

\begin{tabular}{|c|c|c|}
\hline $\begin{array}{lr}\mathrm{RCC} 8 & \text { relation } \\
\text { between } \mathrm{p} \text { and } \mathrm{q}\end{array}$ & \begin{tabular}{|lr} 
RCC8 & relation \\
MBRs p' and q'
\end{tabular} & RCC8 relation between p" and q' \\
\hline$\overline{\mathrm{DC}}(\mathrm{p}, \mathrm{q})$ & 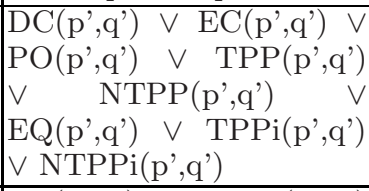 & 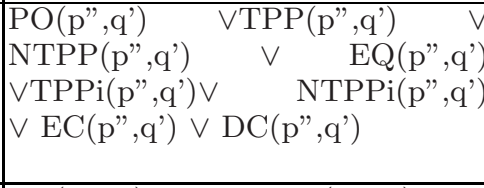 \\
\hline$\overline{\mathrm{EC}}(\mathrm{p}, \mathrm{q})$ & 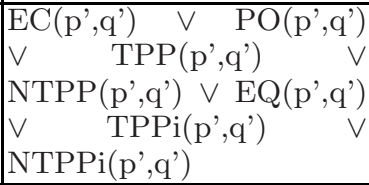 & $\begin{array}{llll}\operatorname{EC}\left(\mathrm{p}^{\prime \prime}, \mathrm{q}^{\prime}\right) & \vee & \operatorname{PO}\left(\mathrm{p}^{\prime \prime}, \mathrm{q}^{\prime}\right) & \vee \\
\operatorname{TPP}\left(\mathrm{p}^{\prime \prime}, \mathrm{q}^{\prime}\right) & \vee & \operatorname{NTPP}\left(\mathrm{p}^{\prime \prime}, \mathrm{q}^{\prime}\right) & \vee \\
\operatorname{EQ}\left(\mathrm{p}^{\prime \prime}, \mathrm{q}^{\prime}\right) & \vee & \operatorname{TPPi}\left(\mathrm{p}^{\prime \prime}, \mathrm{q}^{\prime}\right) & \vee \\
\operatorname{NTPPi}\left(\mathrm{p}^{\prime}, \mathrm{q}^{\prime}\right) & & \end{array}$ \\
\hline$\overline{\mathrm{PO}(\mathrm{p}, \mathrm{q})}$ & 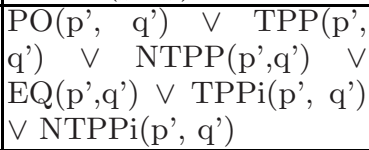 & $\begin{array}{l}\operatorname{PO}\left(p^{\prime \prime}, q^{\prime}\right) \vee \vee \operatorname{TPP}\left(p^{\prime \prime}, q^{\prime}\right) \\
\operatorname{NTPP}\left(p^{\prime \prime}, q^{\prime}\right) \vee \operatorname{EQ}\left(p^{\prime \prime},\right. \\
\left.q^{\prime}\right) \\
\operatorname{TPPi}\left(p^{\prime \prime}, q^{\prime}\right) \vee \operatorname{NTPPi}\left(p^{\prime \prime}, q^{\prime}\right)\end{array}$ \\
\hline$\overline{\mathrm{TPP}}(\mathrm{p}, \mathrm{q})$ & $\begin{array}{l}\text { TPP(p', q') } \vee \operatorname{NTPP}\left(p^{\prime},\right. \\
\left.q^{\prime}\right) \vee \operatorname{EQ}\left(p^{\prime}, q^{\prime}\right)\end{array}$ & 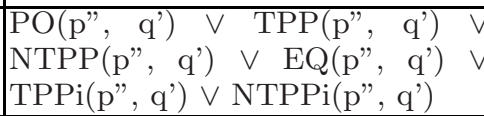 \\
\hline$\overline{\operatorname{NTPP}}(\mathrm{p}, \mathrm{q})$ & NTPP(p', q') & $\begin{array}{l}\operatorname{PO}\left(p^{\prime \prime}, q^{\prime}\right) \vee V \quad \operatorname{TPP}\left(p^{\prime \prime}, q^{\prime}\right) \\
\operatorname{NTPP}\left(p^{\prime \prime}, q^{\prime}\right) \vee V \operatorname{EQ}\left(p^{\prime \prime}, q^{\prime}\right) \\
\operatorname{TPPi}\left(p^{\prime \prime}, q^{\prime}\right) \vee \operatorname{NTPPi}\left(p^{\prime \prime}, q^{\prime}\right)\end{array}$ \\
\hline$\overline{\mathrm{TPPi}}(\mathrm{p}, \mathrm{q})$ & \begin{tabular}{|l}
$\mathrm{EQ}\left(\mathrm{p}^{\prime}, \mathrm{q}^{\prime}\right) \vee$ \\
$\vee \operatorname{TPP}\left(\mathrm{p}^{\prime}, \mathrm{q}^{\prime}\right)$ \\
$\vee \operatorname{NTP}\left(\mathrm{p}^{\prime}, \mathrm{q}^{\prime}\right)$
\end{tabular} & $\begin{array}{l}\text { EQ(p", q") } \quad \vee \\
\left.\text { NTPPi(p", } q^{\prime}\right)\end{array}$ \\
\hline NTPPi $(p, q)$ & NTPPi(p', q') & NTPPi(p", q') \\
\hline $\mathrm{EQ}(\mathrm{p}, \mathrm{q})$ & $\mathrm{EQ}\left(\mathrm{p}^{\prime}, \mathrm{q}^{\prime}\right)$ & $\begin{array}{llll}\text { EQ(p", q') } \vee & \text { TPPi(p", } \\
\text { NTPPi(p", q') } & \vee \\
\end{array}$ \\
\hline
\end{tabular}


Based on the above mapping relationship and the R-tree, the candidate MBR combinations can be retrieved efficiently, and then a refinement step is needed to derive the spatial relations among the real spatial objects that the MBRs enclose, which means that the spatial relation between $\mathrm{p}$ and $\mathrm{q}$ should be derived from the spatial relation between p' and q'. From the spatial relation between two MBRs, we can derive several possible spatial relations or only one definite spatial relation between two real spatial objects that the MBRs enclose. In the former case the complex geometry computation will be applied whereas it will be omitted in the latter case. For example, given the spatial relation $\operatorname{NTPPi}\left(p^{\prime}\right.$, $\left.q^{\prime}\right)$, we can derive $\mathrm{DC}(\mathrm{p}, \mathrm{q}) \vee \mathrm{EC}(\mathrm{p}, \mathrm{q}) \vee \operatorname{PO}(\mathrm{p}, \mathrm{q}) \vee \operatorname{NTPPi}(\mathrm{p}, \mathrm{q}) \vee \operatorname{TPPi}(\mathrm{p}$, $\mathrm{q})$, the geometry computation must be adopted to ascertain the spatial relation between p and q. But if we know the spatial relation $\mathrm{DC}\left(\mathrm{p}^{\prime}, \mathrm{q}^{\prime}\right)$, then spatial relation $\mathrm{DC}(\mathrm{p}, \mathrm{q})$ can be derived directly.

\subsection{Direction Mapping Relationships}

According to Goyal and Egenhofer's cardinal direction model[?], there are 9 atomic cardinal direction relations $(\mathrm{O}, \mathrm{S}, \mathrm{SW}, \mathrm{W}, \mathrm{NW}, \mathrm{N}, \mathrm{NE}, \mathrm{E}, \mathrm{SE})$ (see Fig 2) and totally 218 cardinal direction relations for non-empty connected regions in the Euclidean space $\Re^{2}$ (illustrated by $3 \times 3$ matrix, see Fig [3] [8].

There are 36 cardinal direction relations for the non-empty and connected regions'MBRs: O, S, SW, W, NW, N, NE, E, SE, S:SW, O:W, NW:N, N:NE, O:E, S:SE, SW:W, O:S, E:SE, W:NW, O:N, NE:E, S:SW:SE, NW:N:NE, O:W:E, O:S:N,SW:W:NW,NE:E:SE, O:S:SW:W, O:W:NW:N, O:S:E:SE, O:N:NE:E, O:S:SW:W:NW:N, O:S:N:NE:E:SE, O:S:SW:W:E:SE, $\mathrm{O}: \mathrm{W}: \mathrm{NW}: \mathrm{N}: \mathrm{NE}: \mathrm{E}, \mathrm{O}: \mathrm{S}: \mathrm{SW}: \mathrm{W}: \mathrm{NW}: \mathrm{N}: \mathrm{NE}: \mathrm{E}: \mathrm{SE}($ see Fig 4 ). This kind of cardinal

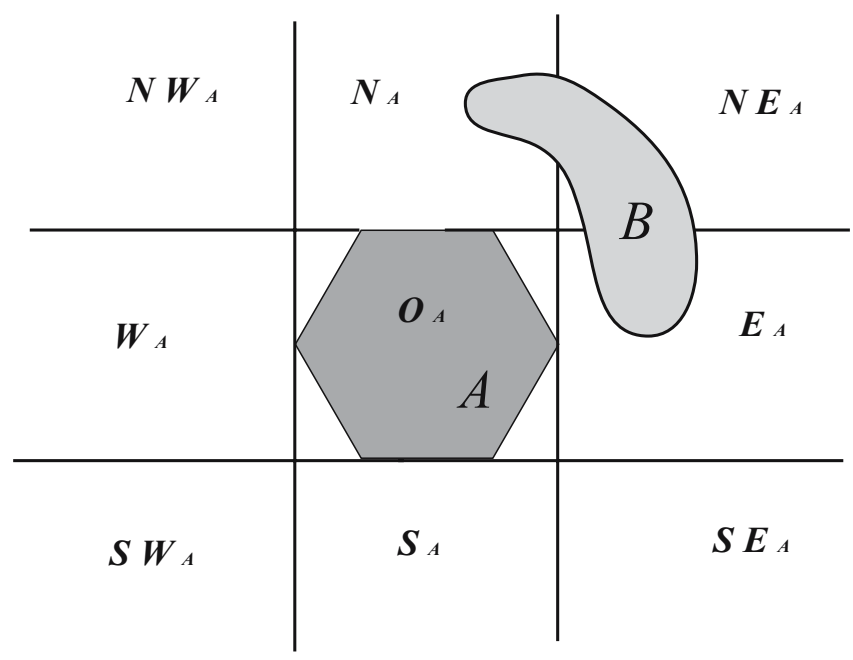

Fig. 2. Capturing the cardinal direction relation between two polygons, A and B, through the projection-based partitions around A as the reference object 


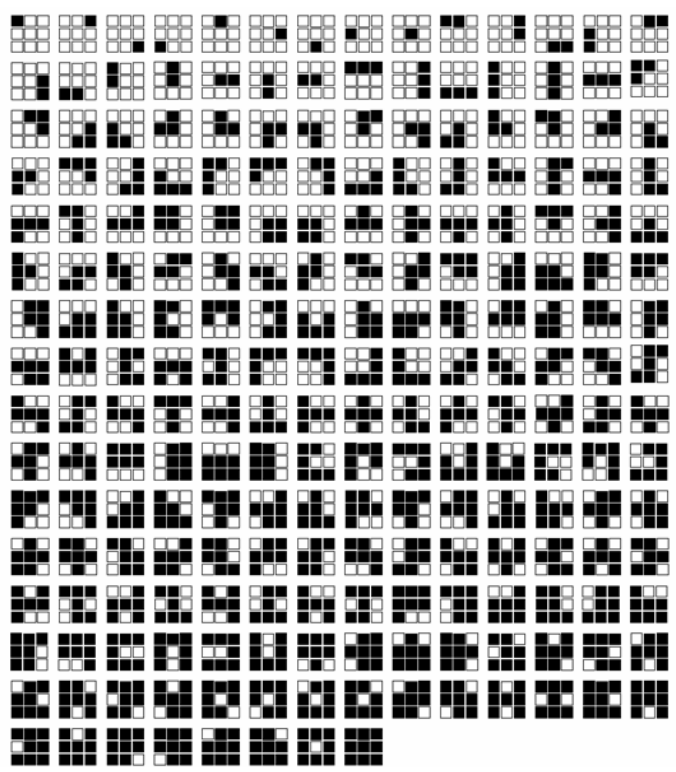

Fig. 3. 218 cardinal direction relations between two non-empty and connected regions 8

direction relation has the rectangle shape, so it is also named rectangle direction relation, otherwise it is called non-rectangle direction relation.

In the following, we study the mapping relationships between the cardinal direction relations for real spatial objects and the cardinal direction relations for the corresponding MBRs. First of all, we give a definition as follows.

Definition 1. a cardinal direction relation $R$ contains another cardinal direction relation $R$ ', if all the atomic relations in $R$ ' also exist in $R$.

The mapping relationships from the cardinal direction relations for real spatial objects to the ones for their MBRs can be described using the following theorems.

Theorem 1. if the cardinal direction relation between the real spatial objects $p$ and $q$ is rectangle direction relation $R$ (see Fig 4 ), the cardinal direction relation between their MBRs $p^{\prime}$ and $q^{\prime}$ is also $R$; if the cardinal direction relation between the real spatial objects $p$ and $q$ is non-rectangle direction relation $R$, the cardinal direction relation between their MBRs $p$ ' and q' is the rectangle direction relation $R$ ' in Fig 4 which contains relation $R$ and has the minimum area.

Theorem 1 can be derived by combining Fig 3 and Fig 4 Assume that the cardinal direction relation between two real spatial objects $p$ and $q$ is $\mathrm{N}: \mathrm{NW}: \mathrm{W}$ which obviously is not rectangle direction relation, from Fig 4 the rectangle direction relation that contains $\mathrm{N}: \mathrm{NW}: \mathrm{W}$ and has the minimum rectangle area is $\mathrm{O}: \mathrm{W}: \mathrm{NW}: \mathrm{N}$, so the cardinal direction relation between two MBRs p' and q' is $\mathrm{O}: \mathrm{W}: \mathrm{NW}: \mathrm{N}$. 


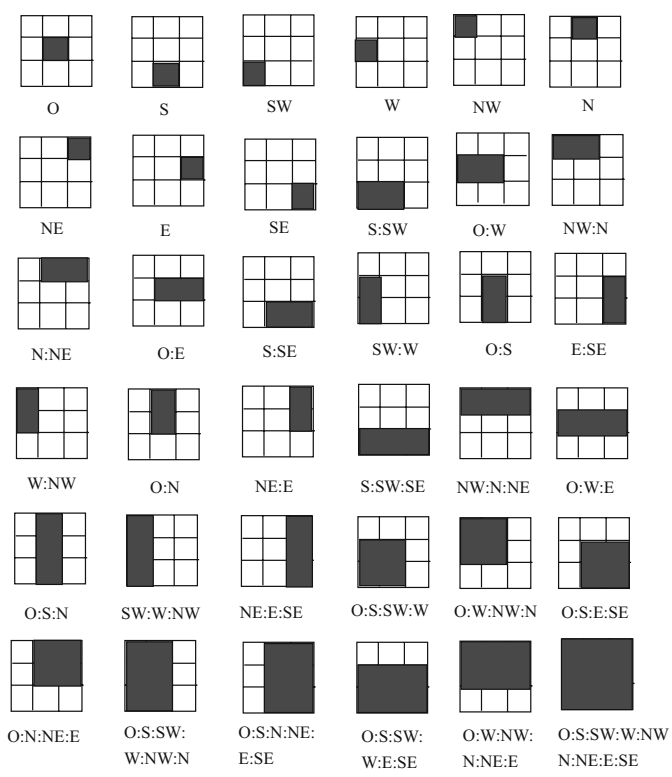

Fig. 4. 36 cardinal direction relations for MBRs

Similarly the mapping relationships from the cardinal direction relations for MBRs to the ones for the possible real spatial objects can be described as follows.

Theorem 2. if the cardinal direction relation $R$ between two MBRs $p$ ' and $q$ ' contains no more than 3 atomic cardinal direction relations (including 3 ), the corresponding cardinal direction relation between the real spatial objects $p$ and $q$ is also $R$; otherwise, the possible cardinal direction relations between $p$ and $q$ will be the subsets of relation $R$ which can be transformed to relation $R$ when $p$ and $q$ are approximated by $p^{\prime}$ and $q$ '.

For example, if the cardinal direction relation between two MBRs is S:SW:SE(including three atomic relations:S,SW,SE), then the cardinal direction relation between the corresponding two real spatial objects definitely is $\mathrm{S}: \mathrm{SW}: \mathrm{SE}$. If the cardinal direction relation between two MBRs is O:S:SW:W, the possible cardinal direction relations between two real spatial objects include $\mathrm{O}: \mathrm{W}: \mathrm{SW}, \mathrm{W}: \mathrm{O}: \mathrm{S}, \mathrm{SW}: \mathrm{S}: \mathrm{O}, \mathrm{SW}: \mathrm{S}: \mathrm{W}$ and $\mathrm{O}: \mathrm{S}: \mathrm{SW}: \mathrm{W}$.

Given the cardinal direction relation between the MBRs p' and q', the cardinal direction relation between p", which is the parent node of p' in R-tree, and q' can be described using the following theorem.

Theorem 3. if the cardinal direction relation between MBRs $p^{\prime}$ and $q$ ' is $R$, the possible cardinal direction relations between $p$ " and q' are the rectangle direction relations containing $R$. 
For example, if the cardinal direction relation between p' and q' is O:S:SW:W, the possible cardinal direction relations between p" and q' will be O:S:SW:W, O:S:SW:W:NW:N, O:S:SW:W:E:SE and O:S:SW:W:NW:N:NE:E:SE.

\section{Conclusions}

This paper has studied the spatial configuration information retrieval problem which includes the mapping relationship among the spatial relations (topological and directional relations) for real spatial objects, the corresponding spatial relations for the corresponding MBRs and the corresponding spatial relations between intermediate nodes and the MBRs in R-tree. Based on these results, search algorithms can be designed to solve the spatial configuration retrieval problems. The research work of this paper is valuable for the information retrieval system related to spatial data.

\section{References}

1. Bergman, L. Castelli, V., Li C-S. Progressive Content-Based Retrieval from Satellite Image Archives. D-Lib Magazine, October 1997. http://www.dlib.org/dlib/october97/ibm/10li.html.

2. Gupta A., Jain R. Visual Information Retrieval. Communications of ACM, May 1997, 40(5): 70-79.

3. Orenstein, J. A. Spatial Query Processing in an Object-Oriented Database System. In: Proc. Of the 1986 ACM SIGMOD international conference on Management of data, 1986, pages 326-336.

4. Guttman, A. R-trees: A Dynamic Index Structure for Spatial Searching. In: Proc. Of ACM SIGMOD, 1984, pages 47-57.

5. Timos K. Sellis, Nick Roussopoulos, Christos Faloutsos. The R+-Tree: A Dynamic Index for Multi-Dimensional Objects. In: Proceedings of 13th International Conference on Very Large Data Bases, September 1-4, 1987, Brighton, England, pages 507-518.

6. Norbert Beckmann, Hans-Peter Kriegel, Ralf Schneider, Bernhard Seeger. The R*tree: An Efficient and Robust Access Method for Points and Rectangles. In: Proceedings of the ACM SIGMOD, 1990, pages 322-331.

7. D. A. Randell, Z. Cui and A. G. Cohn. A Spatial Logic Based on Regions and Connection. In: Proc. 3rd Int. Conf. on Knowledge Representation and Reasoning, Morgan Kaufmann, San Mateo, 1992, pages 165-176.

8. S.Cicerone and P. Di Felice. Cardinal directions between spatial objects: the pairwise-consistency problem. Information Sciences. 2004, 164(1-4): 165-188. 